\title{
Fontes e redes de informação na produção cafeeira do Cerrado Mineiro, MG
}

Information sources and networks in coffee production of Cerrado Mineiro, MG

\section{Sources et réseaux d'information dans la production de café dans le Cerrado Mineiro, MG}

Fuentes y redes de información en la producción de café en el Cerrado Mineiro, MG

\author{
Douglas Ken Nagai* \\ Giuliana Aparecida Santini Pigatto* \\ Timóteo Ramos Queiroz*
}

Recebido em 26/04/2016; revisado e aprovado em 22/06/2016; aceito em 10/10/2016

DOI: http:/ / dx.doi.org/10.20435/1984-042X-2016-v.17-n.4(09)

\begin{abstract}
Resumo: O objetivo foi analisar as fontes de informação para a inovação utilizadas no processo de obtenção do registro de Denominação de Origem (DO) na região do Cerrado Mineiro, em termos de atores e redes de informação. Foram aplicados estudos de caso com onze produtores rurais do município de Patrocínio, MG. Os resultados demonstraram que alguns produtores que comercializam com o selo de origem ocupam de fato um papel central, tanto na frequência de busca da informação, como em ser fonte de informação por parte de outros produtores.
\end{abstract}

Palavras-chave: denominação de origem; produção de café; inovação.

Abstract: The objective was to analyze the sources of information for innovation used in the process of obtaining the Designation of origin register in the Cerrado Mineiro region in terms of actors and information networks. Case studies were applied with eleven farmers of Patrocínio city, MG. The results showed that some producers who marketing with origin label occupy a central role, both in the frequency of information search as in source of information by other producers.

Key words: protected designation of origin; coffee production; innovation.

Résumé: L'objectif était d'analyser les sources d'information pour l'innovation utilisée dans le processus d'obtention de l'enregistrement Appellation d'origine dans la région du Cerrado Mineiro en termes d'acteurs et de réseaux. études de cas ont été appliquées avec onze agriculteurs de la municipalité de Patrocínio, MG. Les résultats ont montré que certains producteurs qui commercialisent avec le label d'origine occupent un rôle central tant dans la fréquence des informations l'innovation en tant que source d'information par d'autres producteurs.

Mots-clés: appellation d'origine; la production de cafe; l'innovation.

Resúmen: El objetivo fue analizar las fuentes de información para la innovación utilizado en el proceso de obtener el registr Denominación de Origen en la región del Cerrado Mineiro en términos de actores y redes. Estudios de casos se aplicaron con once agricultores en el municipio de Patrocinio, MG. Los resultados mostraron que algunos productores que comercializan con la etiqueta de origen ocupar un papel central tanto en la frecuencia de búsqueda de información como fuente de información por otros productores.

Palabras clave: denominación de origen; la producción de café; la innovación.

\section{INTRODUÇÃO}

O café possui alta relevância na economia e sociedade brasileira desde sua implantação, no século XVIII, época na qual o Brasil ainda operava nos moldes de uma colônia. Implantado primeiramente nos estados do Pará e Maranhão e, depois, expandido ao longo de sua história para quinze estados, com destaque para Minas Gerais, o café mostrou grande adaptabilidade ao clima e solo local, e se tornou um importante item da pauta comercial brasileira (BRASIL, 2014).

Essa trajetória tornou o Brasil maior produtor de café mundial, seguido de Vietnã, Indonésia e Colômbia. Dados demonstram que o Brasil é responsável

\footnotetext{
*Universidade Estadual Paulista “Júlio de Mesquita Filho" (Unesp), Tupã, São Paulo, Brasil.
} 
por cerca de $30 \%$ da produção mundial de café desde 2001 e produziu aproximadamente cinquenta milhões de sacas, em 2014 (COMPANHIA NACIONAL DE ABASTECIMENTO [CONAB], 2015). O café atualmente é o quinto produto mais relevante na pauta de exportações do agronegócio brasileiro, com aproximadamente $5 \%$ do valor total exportado, sendo este menor apenas que o complexo da soja, carnes, setor sucroenergético e produtos florestais (BRASIL, 2014).

No contexto nacional, o estado de Minas Gerais é o maior produtor, tendo produzido cerca de 1,33 milhões de toneladas de café em 2015 (52\% do nacional), sendo também responsável por mais da metade da área plantada, seguido de Espírito Santo, São Paulo, Bahia e Paraná. Entre as regiões que compõem o estado de Minas Gerais, a do Cerrado Mineiro, segundo Ortega e Jesus (2012), receberam diretamente os benefícios originários da Revolução Verde, que auxiliou a produção local de maneira eficiente, por meio da introdução de inovações tecnológicas ao longo de sua trajetória histórica. No que se refere às características naturais e climáticas do Cerrado Mineiro, Ortega e Jesus (2011) afirmam que a região é propícia para produção de café com qualidade.

Segundo Mafra (2008), a partir da década de 1990, o café do Cerrado passou por um processo de inovação organizacional e institucionalizou-se como uma rede organizacional aberta, por meio da criação do Conselho das Associações dos Cafeicultores do Cerrado (CACCER), que atualmente transformou-se em Federação dos Cafeicultores do Cerrado. Segundo o autor, a criação do CACCER foi realizada como uma forma de iniciativa local com o objetivo de valorizar e melhorar a qualidade do café na região e significou um maior nível de cooperação institucional em relação a outras regiões produtoras.

Outro aspecto importante relacionado à região do Cerrado Mineiro foi a mudança de status no produto café, obtida pela Indicação Geográfica (IG). A produção no Cerrado Mineiro entrou com seu primeiro pedido de IG em 1995, com o objetivo de diferenciar seu produto e acessar mercados internacionais, bem como aumentar o preço comercializado (MAFRA, 2008). Segundo Mafra (2008), apesar da valorização da região em si provida pela IG, esta também trouxe ao produto uma grande melhoria de marketing. O café passou a ser respeitado não só internacionalmente, mas também pelos produtores, que passaram a considerá-lo como um produto de alto nível. Esse reconhecimento fomentou ainda mais a participação ativa dos produtores em organizações, como associações e a Federação do Cerrado, e cooperativa credenciada para exportação do café da região, a Expocaccer.

Guimarães Filho (2013) precisa também que as IGs são um tipo de registro que garante a proteção e identidade da cultura local. As IGs concedidas e registradas pelo Instituto Nacional de Propriedade Industrial (INPI, 2013) são classificadas em: i) Indicação de Procedência (IP) - é o nome geográfico de um país, cidade, região ou de uma localidade que se tornou conhecida como centro de produção, fabricação ou extração de determinado produto ou prestação de determinado serviço. É importante lembrar que, no caso da Indicação de Procedência, é necessária a apresentação de documentos que comprovem que o nome geográfico seja conhecido como centro de extração, produção ou fabricação do produto ou prestação do serviço; ii) Denominação de Origem (DO) - é o nome geográfico de país, cidade, região ou localidade de seu território, que designe produto ou serviço cujas qualidades ou características se devam exclusiva ou essencialmente ao meio geográfico, incluídos fatores naturais e humanos. Na solicitação da IG de Denominação de Origem, deverá ser apresentada também 
a descrição das qualidades e as características do produto ou serviço que se destacam, exclusiva ou essencialmente, por causa do meio geográfico, ou de fatores naturais e humanos.

Dentre as regiões que obtiveram o registro de DO no Brasil, no tocante à produção de café, a do Cerrado Mineiro foi a única a obtê-lo em 2013. Após a implantação da DO, o INPI (2013) estabeleceu que o café produzido na região do Cerrado Mineiro devesse possuir uma série de qualidades referentes ao sabor, aroma e qualidade do produto, sendo que, para se manterem tais qualidades, são necessárias técnicas avançadas de cultivo, plantio e manejo das plantas.

Tais técnicas exigiram potenciais mudanças na forma de gestão, uso de recursos, informação e relação com os demais atores envolvidos na atividade cafeeira. Desse modo, o objetivo principal deste artigo é o de analisar as fontes de informação para a inovação utilizadas nesse processo de obtenção do registro de $\mathrm{DO}$ na região, em termos de atores e redes de informação. A análise é focada nos produtores rurais de café que estão na região do Cerrado Mineiro (seja de produtores que comercializam ou não com o selo de origem), por meio da avaliação de três redes sociais envolvendo os objetivos, a frequência de uso e o nível de confiança nas fontes de informação para a inovação.

Em face de atingir os objetivos, este artigo está estruturado em seis seções. Após a introdução são apresentadas, nas seções dois e três, as contribuições teóricas acerca dos temas de fontes de conhecimento e redes sociais. Seguindo à seção quatro, são trabalhados os métodos empregados na pesquisa, dando-se sequência, na seção cinco, aos resultados e discussões. Encerrando o trabalho, são apresentadas as considerações finais na sexta seção e, posteriormente, as referências para embasamento teórico.

\section{FONTES DE INFORMAÇÃO PARA A INOVAÇÃO NOS SISTEMAS AGRÍCOLAS E SEUS CONDICIONANTES}

O uso das fontes de informação, para Jespersen et al. (2014), está ligado às redes de interações sociais para compartilhar, registrar e utilizar as informações nos sistemas agrícolas. Essas redes podem ser expressas informalmente, na forma de grupos de produtores ou demais atores e, formalmente, na forma de instituição ou rede social digital.

Segundo Poppe (2012), o processo linear de transferência de tecnologia e geração de inovação (de cientistas para usuários) é ultrapassado, devendo este ser substituído por um modelo interativo de rede, que inclua agentes ligados à produção, educação, adaptação e orientação. Essa substituição se dá ao fato de novos conhecimentos estarem sendo gerados por produtores, pesquisadores e também por empresas privadas.

Holster et al. (2012) afirmam que o uso do conhecimento nos sistemas agrícolas, para uma maior ou menor aderência à tecnologia, está baseado em elementos como a presença de computadores na fazendas, acesso a internet, uso de um sistema informatizado de gestão, a quantidade e uso de telefonia móvel. Esses elementos, segundo os autores, ocorrem em diversos níveis e, simultaneamente, demonstram o acesso à facilidade e organização do uso das fontes de informação nas fazendas. Os autores analisaram também que a falta de conexões de banda larga e potentes para uso da internet são um fator limitante à melhor interatividade das propriedades rurais.

O uso das fontes de informação também depende de fatores determinados pelas características e formas de interação entre os agentes envolvidos no processo produtivo em questão. Nesse caso, devem ser considerados fatores como idade dos usuários da informação e seus níveis 
educacionais, questões culturais, disponibilidade de informação e facilidade em se obter ferramentas para o uso da informação. Outro aspecto importante são suas formas de comunicação com os demais atores, expresso pelo nível de formalidade e burocratização, baseado na existência de registros ou atas, no caso de instituições ou registros rastreáveis de informações, no caso de uma comunicação digital (JESPERSEN et al., 2014).

Nessa perspectiva, um dos atores destacados por Docks, Tisenkopfs e Bock (2012) é o consumidor final, pois este age como um corretor de informações que fornece uma resposta a cada produto ou serviço lançado ao mercado. Os autores ressaltam que as informações sinalizadas pelos consumidores finais devem ser transmitidas até os produtores rurais, para que possam reformular constantemente suas atividades e gerar potencial inovador.

Outro ator de importância como fonte de informação são os institutos de pesquisa, analisados por Beaulieu (2013) e Cooke, Uranga e Etxebarria (1997) como uma forma de se catalisar e gerar inovações para um setor ou território, e fornecem opções nas formas de produção e organização dos demais agentes envolvidos em uma atividade fim. Entretanto nem toda inovação gerada por um instituto de pesquisa é utilizada diretamente pelos produtores rurais, seja por falta de recursos para investimentos, seja por problemas de se obter informações acerca da inovação em questão.

No Brasil, deve-se destacar a importância dos institutos públicos de pesquisa para a inovação, como a Empresa Brasileira de Pesquisa Agropecuária (Embrapa), responsável pelo desenvolvimento de novos conhecimentos e inovações na agropecuária. Entre as culturas abordadas pela Embrapa estão a soja, hortaliças, milho, gado e café (EMBRAPA, s.d.).

Outro instituto importante como fonte de informação no Brasil é a Empresa de Assistência Técnica e Extensão Rural (EMATER), que é responsável por fornecer serviços de assistência aos produtores, como programas técnicos, organizacionais e comerciais. No caso de Minas Gerais, a unidade atuante é a EMATERMG, vinculada à secretaria Estadual de Agricultura, Pecuária e Abastecimento (EMATER-MG, s.d.).

SegundoaOrganisationForEconomic Co-Operation And Development (OECD, 2005), há uma série de fontes de informações para a geração de inovações e transferência de conhecimento, e essas fontes podem ser classificadas em: fontes abertas de informação, fontes pagas de informação e fontes originárias de parcerias entre os atores envolvidos em uma atividade fim. As fontes de informação abertas podem ser acessadas de maneira gratuita, não possibilitando gerar patentes nem outras formas de proteção. As fontes pagas geralmente referem-se à aquisição de uma nova fonte de conhecimento, na qual não há necessariamente cooperação com o agente que forneceu a fonte. Tais fontes podem ser desde a aquisição de um novo ativo, como maquinário ou software, como também a contratação de um especialista para a realização de um serviço específico.

Quanto às fontes de informação providas por meio de parcerias, estas fornecem maior impulso à ocorrência de inovações, uma vez que há a ativa cooperação para o desenvolvimento e manutenção das informações e conhecimento entre os agentes, em uma relação sinérgica. Nessa categoria, incluem-se as alianças estratégicas, convênios entre empresas, projetos conjuntos e pesquisas que envolvam a participação do mercado consumidor (OECD, 2005). Também para Freeman (1991), além de fatores ligados aos agentes empreendedores, as inovações também se originam de informações distribuídas em redes para inovações. As redes podem ocorrer de diversas maneiras para intercambiar informações, por 
meio de contratos e alianças estratégicas, fomentos governamentais, parcerias com instituições que trabalham com pesquisa e redes informais capazes de gerar informações.

Essas fontes de informação também podem se manifestar como uma rede do uso da informação por parte dos produtores rurais, a qual pode condicionar seu comportamento e estar combinada com fatores exógenos para a decisão sobre adotar ou não uma inovação (ORESZCZYN; LANE; CARR, 2010).

Entretanto o uso da informação também deve contemplar a difusão da informação transmitida aos consumidores participantes de um processo de inovações de Marketing e divulgação, pois, no caso do ambiente de produção rural, a participação dos consumidores finais, muitas vezes, é condicionante do sucesso da inovação (BRUNORI; ROSSI; GUIDI, 2012).

\section{REDES SOCIAIS COMO FORMA DE REPRESENTAÇÃO DAS FONTES DE INFORMAÇÃO E USO DO CONHECIMENTO}

A forma como os produtores rurais se organizam podem ser demonstradas por meio das redes sociais. As redes são uma forma de demonstrar o capital social que envolve diferentes agentes em uma atividade fim, entre elas indivíduos, entidades e formas de relacionamentos (AHUJA; SODA; ZAHEER, 2012).

Nascimento, Jimenez e Campomar (2014) analisaram que as redes sociais são consideradas uma importante fonte de informação para decisão de adquirir um bem ou serviço. Segundo os autores, as redes proporcionam ao consumidor a chance de comparar atributos, benefícios, garantias e experiências de outros usuários do produto ou serviço em questão.

Schaefer (2008) argumentou que o processo de transferência por meio da interação entre os agentes em uma rede social pode ocorrer de maneira esgotável ou contínua. No caso da transferência esgotável, um mesmo nó não poderia fornecer algo que já foi transferido, como no caso dos bens materiais. Por exemplo, um mesmo agente que já fornecera cinco reais a outro agente não poderia fornecer os mesmos cinco reais, a não ser que os cinco reais a ele retornassem. No caso das transferências contínuas, o que for transferido é replicável e não se esgota caso um agente o transfira. No caso da transferência de conhecimento, um agente pode fornecer o mesmo conhecimento para múltiplos receptores de maneira inesgotável.

Essas redes também não são estáticas e tendem a se modificar com o tempo, seja com mudanças nas relações entre os nós, seja pela adesão ou abandono de membros. As redes também são sujeitas a alterações devido a fatores exógenos, como a conjuntura econômica ou ciclos de vida dos produtos, e tecnologias condicionantes das relações sociais. Tais alterações levam a um potencial gerador de inovações, tanto pela maturidade da rede ou por novas necessidades emergentes (AHUJA; POLIDORO; MITCHELL, 2009).

O uso do conhecimento para se gerar inovações também recebe contribuições dos estudos ligados às redes sociais, uma vez que tais estudos assumiram que as inovações não ocorriam na forma de globalização tecnológica, mas sim, localmente (ou nacionalmente), por meio da interação entre diferentes atores econômicos, políticos e sociais (CASSIOLATO; LASTRES, 2005). Cassiolato e Szapiro (2003) também analisaram que o uso intensivo da informação, o aprendizado coletivo e a identidade social e cultural são elementos que fazem parte das dimensões de uma rede social. Os autores também afirmam que essas dimensões são dependentes de políticas (públicas ou privadas), em nível local, para o desenvolvimento dos agentes e do institucional (delimitado pela rede), para se atingir os mercados-alvo desejados, sejam estes locais ou internacionais. 
No que tange ao dinamismo de uma rede social, Granovetter (2007) analisou que existe uma sobreposição entre a racionalidade econômica clássica e as relações sociais, de maneira contínua e indissociável. Tal sobreposição influencia na tomada de decisão individual de cada membro da rede. Os agentes que fazem parte de uma rede estão imersos ${ }^{1}$ em uma relação social, entre elas a familiar, a profissional e cotidiana; e também no racionalismo econômico, influenciado pelo comportamento que objetiva o benefício individual ou estratégia preestabelecida. Em ambos os casos, o autor afirma que a forma como uma rede ocorre está atomizada em decisões individuais dos membros. No caso das relações sociais, a rede social influencia e interioriza o comportamento de cada indivíduo ou entidade membro da rede. Já no caso da racionalidade econômica, os indivíduos tomariam decisões individuais baseadas no benefício próprio.

As relações sociais, por meio da confiança entre os membros, também ajudam o grupo a compartilhar normas, seja para se atingir um benefício comum a todos, seja para sancionar irregularidades realizadas pelos membros. As redes, muitas vezes, também ajudam a solucionar conflitos sem aporte jurídico; uma vez que algum indivíduo cometa atos ligados a má-fé ou oportunismo, ele perderia benefícios coletivos e a confiança dos demais membros (ALBAGLI; MACIEL, 2002).

Para Granovetter (2007), as redes sociais são mutáveis mediante os atos de má-fé, oportunismo ou desconfiança entre os membros, pois, caso o cenário de relações sociais esteja em caráter duvidoso ou insatisfatório, os membros de uma rede tendem a buscar outros grupos

\footnotetext{
${ }^{1}$ A imersão aqui mencionada origina-se da proposta de imersão chamada de embeddedness, a qual pressupõe o fato de as relações sociais serem presentes e constantes no comportamento dos indivíduos e instituições, o que torna equivocado estudá-los individualmente (GRANOVETTER, 2007).
}

negociantes no mercado, estabelecendo novos laços de relacionamento. Por outro lado, quando existe a confiança, redes tendem a ampliar sua estrutura atual, com novos membros que possuam objetivos comuns à rede e tragam benefícios coletivamente.

Para Albagli e Maciel (2004), os ambientes institucionais que possuam uma capacidade social difusa, culturalmente moldada e histórica para que seus membros interajam, sejam estes indivíduos ou entidades, são capazes de gerar e de apropriar-se de novos conhecimentos, bem como de utilizá-los em favor do desenvolvimento local e coletivo.

Os ambientes mais favoráveis à cooperação e confiança ocorrem em locais que possuam interações numericamente expressivas, regulares e com maior complexidade, considerando as relações formais e informais. No caso dos ambientes que possuem relacionamentos escassos e não frequentes, e não há vínculo entre os agentes, a cooperação a atividades conjuntas tende a ser menor (ALBAGLI; MACIEL, 2004).

\section{MÉTODO DE PESQUISA}

Para este trabalho foi utilizada a abordagem qualitativa, delimitada por Dalfovo, Lana e Silveira (2008) como ideal para situações em que os números não servem como indicadores das informações coletadas, mas, sim, palavras e interpretações.

Outro tipo de procedimento escolhido para o desenvolvimento da pesquisa foi o de múltiplos casos, definido por Voss, Tsikriktsis e Frohlich (2002) como adequado para situações na qual há testes de validação da teoria. Assim, foi realizada uma pesquisa de campo, por meio da aplicação de um formulário com sete produtores de café que comercializam com DO e quatro produtores de café sem registro de origem na região do Cerrado Mineiro, especificamente na cidade de 
Patrocínio, MG. A escolha dessa cidade foi baseada não apenas pela presença dos produtores citados anteriormente, mas também por ser a cidade sede da Federação do Cerrado Mineiro. Os estudos foram realizados em março do ano de 2015, mas contemplando-se informações do período de 2012 a 2014.

As informações geradas durante a pesquisa de campo geraram o mapeamento e análise das fontes de informação, por meio do software UCINET 6, permitindo a construção de grafos, tabelas e base de dados para determinar as interações referentes à busca e obtenção de informações para o processo de inovações (BORGATTI; EVERETT; FREEMAN, 2002).

Para analisar as fontes de informação, foi escolhido o modelo de redes sociais, que, segundo Granovetter (2007), são uma forma de interpretação que busca o equilíbrio entre o caráter econômico e social de um local, arranjo ou organização. Essas redes refletem o comportamento de um indivíduo em uma análise não atomizada e incluem representações das relações entre eles.

Os elementos básicos de análise para a rede estiveram pautados em nós, laços e na estrutura da rede, trabalhados por Ahuja, Soda e Zaheer (2012). Os nós representam as unidades de um estudo, podendo ser indivíduos ou organizações representados conforme o objetivo em questão; os laços representam uma forma estabelecida de relacionamento ou interação entre os nós, como confiança ou dependência entre eles. A estrutura da rede determina quais serão as regras que a determinam e o que ela representa em termos de organização dos nós e laços.

\section{RESULTADOS E DISCUSSÕES}

O estudo das redes sociais permitiu demonstrar quais são as fontes de informação de inovação utilizadas pelos produtores rurais. Ao todo, foram identificados onze produtores rurais (A,
B, C, D, E, F, G, H, I, J, K) e onze fontes de informação (de um total de quinze), entre elas organizações, instituições, fontes impressas e digitais de informação. As organizações identificadas foram: Cooperativa EXPOCCACER, Federação dos Cafeicultores do Cerrado, Sindicato Rural de Patrocínio, MG; e as instituições públicas foram: Ministério da Agricultura, Pecuária e Abastecimento (MAPA), EMBRAPA, INPI, Prefeitura de Patrocínio, MG, e Emater Minas Gerais. As demais fontes identificadas foram a internet e as revistas eletrônicas e impressas.

A fim de se analisar como as fontes de informação são utilizadas, foram elaborados três mapas de rede social, a partir da aplicação do software UCINET 6. O primeiro estabeleceu os objetivos do uso das fontes de informação por parte dos produtores rurais; a segunda demonstrou a frequência de uso das fontes de informação; e a terceira, a relação de confiança dos produtores rurais em relação às fontes de informação. As redes formadas podem ser consideradas como egocêntricas, conforme Ahuja, Soda e Zaheer (2012), uma vez que foram entrevistados apenas os produtores agrícolas nesta pesquisa.

De maneira geral, em todas as redes apresentadas, foram estabelecidos dois tipos de nós, sendo um deles os de produtores rurais (grupo de onde partiu a informação da aplicação do formulário) e o outro, das fontes de informação (formada por produtores rurais, organizações, instituições e outras fontes). Em relação à análise dos mapas, a forma de análise da rede foi feita em consonância com os autores Ahuja, Soda e Zaheer (2012), Granovetter (2007) e Ahuja, Polidoro e Mitchell (2009), por meio da contagem dos laços existentes entre os produtores e as fontes de informação para estabelecer ordenamentos entre os nós. 


\subsection{Análise das redes sociais dos produtores rurais baseada nos tipos de informação voltados à inovação}

No que tange aos objetivos do uso das fontes, o mapa da rede social elaborado considerou as possíveis combinações geradas a partir da combinação de quatro objetivos principais. Essas categorias de objetivos foram escolhidas a partir das tipologias das inovações, do Manual de Oslo (OECD, 2005). A categoria outros englobou elementos comerciais, mercadológicos e relativos ao selo da DO.

A figura 1 mostra o mapa de rede social no tocante aos objetivos do uso das fontes de informação por parte dos produtores rurais. Ao visualizar o mapa, é possível perceber que os nós em azul representam as fontes de informação. Entretanto é necessário lembrar que esta é uma rede centrada em produtores rurais, ou seja, os nós que indicam os produtores rurais também são os que consultam as fontes de informação.

Portanto os laços que partem dos produtores foram considerados como uma resposta positiva ao uso de uma fonte de informação; e os laços que chegam aos nós indicam o fato da fonte ser consultada. Logo, não há laços que, por exemplo, partam de uma instituição ou organização, uma vez que, como dito anteriormente, os respondentes dos formulários são todos produtores rurais.

Entre os nós de fontes de informação centrais, baseados no número de laços, foram identificados: o uso da internet, a Cooperativa Expocaccer e a Federação dos Cafeicultores do Cerrado, e três produtores (A, B e $\mathrm{C}$ ). O nó que representa a internet possui 11 laços, sendo considerada o mais central da rede. Os produtores de maneira geral declararam que a internet possui vasta informação em relação à produção de café e novas tecnologias utilizadas no processo produtivo, cabendo a eles selecionar as informações pertinentes que serão utilizadas na gestão da propriedade. A análise dos formulários demonstrou que todos os produtores rurais têm acesso a computadores e a internet em suas residências e, conforme analisado por Holster et al. (2012), auxilia no acesso e organização das informações por parte dos produtores rurais.

Já a Cooperativa Expocaccer e a Federação dos Cafeicultores do Cerrado, ambas com nove laços, são organizações fundamentais para o funcionamento do sistema de produção local de café de origem. A Expocaccer assume um papel de importância comercial e institucional, uma vez que é a cooperativa credenciada para realizar as exportações do café de origem. Já a Federação dos Cafeicultores do Cerrado assume um papel institucional também importante, uma vez que é o órgão responsável pelo credenciamento da região como utilizadora da DO junto ao INPI e também auditora da qualidade do café produzido na região. No caso dessas organizações, as informações fornecidas aos produtores podem ser compreendidas pela categoria outros do mapa da rede social. No caso da Expocaccer, a informação consultada é de natureza comercial, relativa a preço de venda da produção ou referente ao preço para armazenamento da produção nos estoques da cooperativa. No caso da Federação, a informação consultada é referente ao funcionamento da DO, como obtê-la e quais as vantagens oferecidas. 


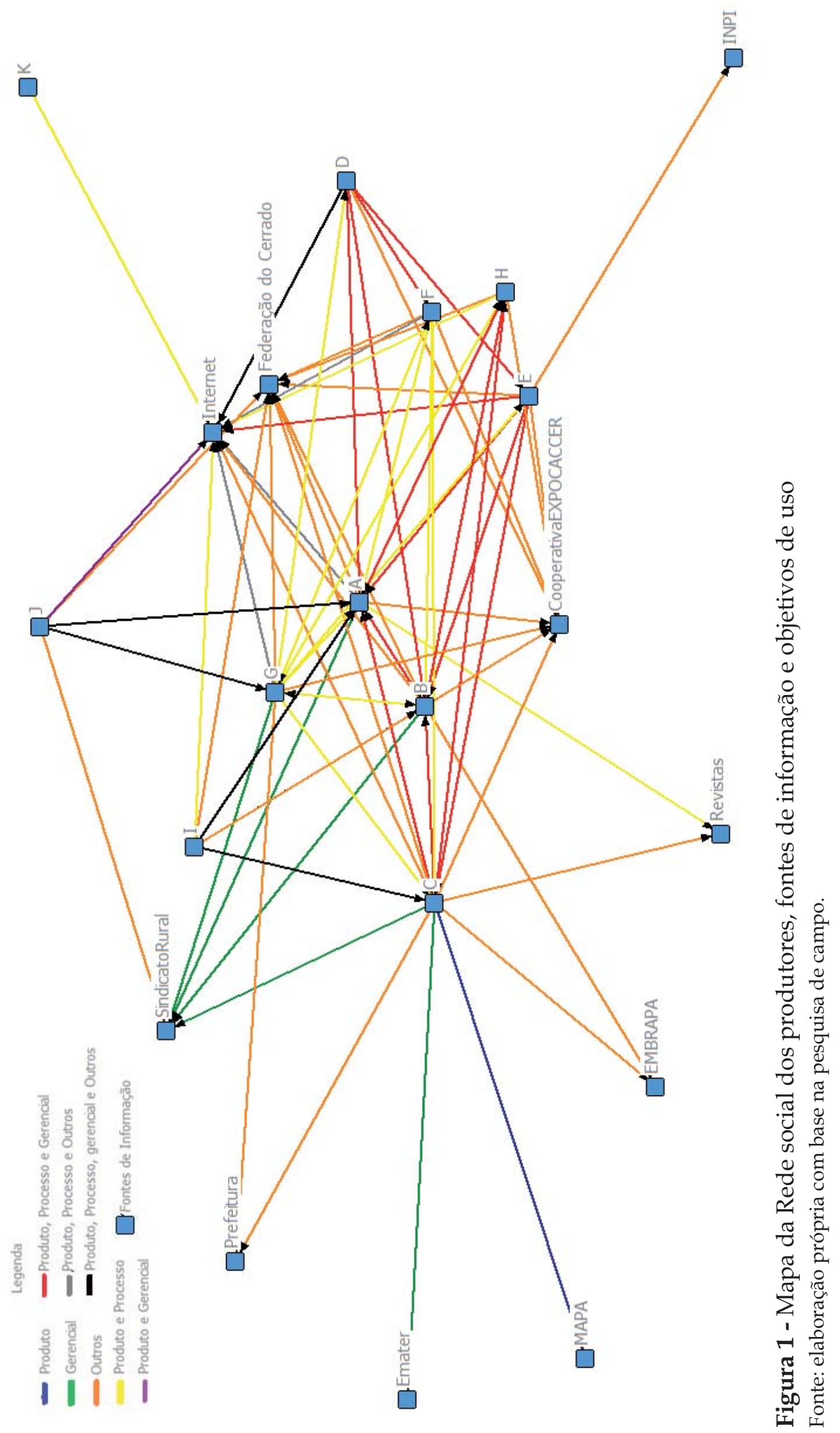


Os nós $\mathrm{A}, \mathrm{B}$ e $\mathrm{C}$, todos produtores que comercializam com a $\mathrm{DO}$, com nove, nove e oito laços respectivamente, são produtores que possuem um ritmo elevado de adoções de inovações em seus processos produtivos. Apesar de as instituições públicas, como o INPI e a Embrapa, não terem obtido pontuação elevada, é necessário ressaltar que ambas possuem informações disponíveis para consulta na Internet. Os produtores de maneira geral declararam utilizar a internet como fonte de informação, mas não especificaram quais portais são acessados com maior frequência. Existe, nesse caso, a potencialidade de as informações desses órgãos estarem sendo acessadas via internet. No caso do INPI, as informações são selecionadas e difundidas por meio da Federação dos Cafeicultores do Cerrado, que é a organização responsável por manter a DO na região.

\subsection{Análise das redes sociais dos produtores rurais baseada na frequência de uso}

A figura 2 mostra o mapa da rede social baseado na frequência de uso das fontes de informação. No caso das fontes de informação utilizadas, a internet e a cooperativa Expocaccer foram as que obtiveram as maiores pontuações (32 e 23 respectivamente), em termos do número de laços e escala de periodicidade/ intensidade de uso (quadro 1). Entretanto uma diferença identificada entre essas duas entidades está no nível de integração que cada uma proporciona. No caso da internet, os produtores buscam informações de maneira independente e individual, não havendo um espaço virtual formal para troca de informações entre os produtores.

No caso da Cooperativa EXPOCACCER, a troca de informações ocorre por meio de encontros presenciais, em reuniões, cursos ofertados ou casualmente, tornando a troca de informações mais coordenada. A EXPOCACCER também ocupa um papel importante como fonte de informação, por ser o órgão integrador para a realização das exportações de café da região.

Entre os produtores rurais, os que obtiveram maior pontuação em termos de fontes de informação foram os A, B e C, com 20, 20 e 15 pontos, no âmbito de frequência de consulta respectivamente (que também foram os três produtores já analisados na subseção anterior como de maior busca por fonte de informação para a inovação). Os produtores com DO também possuem maior pontuação como fonte de informação. Uma exceção é o produtor $\mathrm{H}$, com 11 pontos, que está em transição para comercializar com a DO e ocupa um cargo de liderança em uma associação de pequenos produtores. 


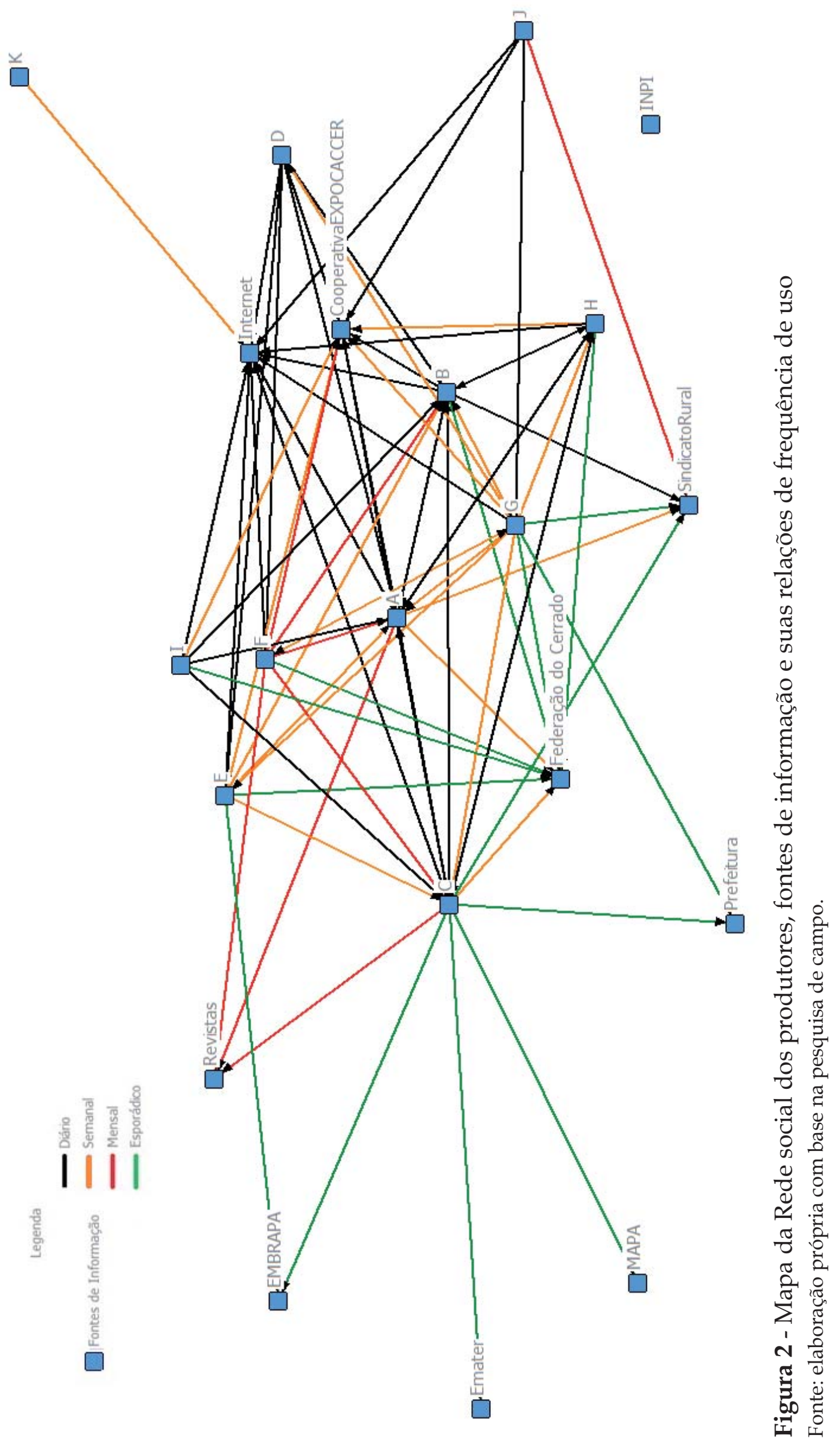


O quadro 1 foi elaborado a fim de se estebelecer um ordenamento baseado na frequência de uso das fontes de informação (quais produtores mais frequentemente acessariam as fontes de informação).

\begin{tabular}{|c|c|c|c|}
\hline Fonte de Informação & Pontuação & Fonte de Informação & Pontuação \\
\hline Internet & 32 & Revistas & 3 \\
\hline Expocaccer & 23 & D (DO) & 2 \\
\hline Produtores A e B (DO) & 20 & EMBRAPA & 1 \\
\hline Produtor C (DO) & 15 & Prefeitura & 1 \\
\hline H (N DO) & 11 & MAPA & 0,5 \\
\hline Federação do Cerrado & 7 & Emater & 0,5 \\
\hline Sindicato Rural & 5 & INPI & 0 \\
\hline Produtor E (DO) & 5 & I (NDO) & 0 \\
\hline Produtor F (DO) & 5 & I (NDO) & 0 \\
\hline Produtor G (DO) & 5 & K (NDO) & 0 \\
\hline
\end{tabular}

Quadro 1 - Ordenamento das fontes de informação em relação à frequência de uso pelos produtores rurais (em ordem decrescente de pontuação quanto à periodicidade do uso da informação)

Fonte: elaboração própria com base na pesquisa de campo.

O quadro 2 mostra o ordenamento dos produtores rurais em relação à frequência do uso das fontes de informação (quais produtores mais frequentemente acessariam as fontes de informação). Assim como no quadro 1, o quadro 2 também utilizou a contagem dos nós e a somatória baseada na frequência (três pontos para o uso diário das fontes, dois para o uso semanal, um para o uso mensal e meio ponto para o uso esporádico).

\begin{tabular}{|c|c|c|c|}
\hline Produtores com DO & Pontuação & Produtores sem DO & Pontuação \\
\hline B & 21,5 & H e I & 14,5 \\
\hline C e G & 20,5 & J & 9 \\
\hline A & 20 & K & 2 \\
\hline D & 18 & & \\
\hline E & 12 & & \\
\hline F & 10,5 & & \\
\hline
\end{tabular}

Quadro 2 - Ordenamento dos produtores rurais em relação à frequência de busca das fontes de informação (em ordem decrescente de pontuação quanto à periodicidade da busca da informação).

Fonte: elaboração própria com base na pesquisa de campo.

De maneira geral, é possível perceber que os produtores que comercializam com a DO possuem uma busca mais intensa e uma maior frequência de uso de fontes de informação para a inovação. É possível perceber também que os cinco produtores que obtiveram a maior pontuação (em frequência de uso, como o A, B, C, D e G) são todos usuários da DO. Entretanto é possível analisar que existem produtores sem DO com pontuação superior a produtores com DO, como no caso dos Produtores $\mathrm{H}$ e I, que possuem pontuação maior que os produtores E e F. Porém os produtores H e I afirmaram estar em transição para começar a comercializar com a DO.
Portanto pode-se inferir que as redes de informação para a inovação são mais efetivas nas produções que comercializam com o registro de DO, todavia existem unidades produtoras sem DO que obtiveram maior frequência de uso de fonte de informação em relação a produtores com $\mathrm{DO}$, apesar do ritmo menos intenso de adoção de inovações.

\subsection{Análise das redes sociais dos produtores rurais baseada na confiança nas fontes de informação}

Para esta etapa da análise entre os produtores rurais, a figura 3 mostra o mapa da rede social baseado na confiança de uso das fontes de informação. 


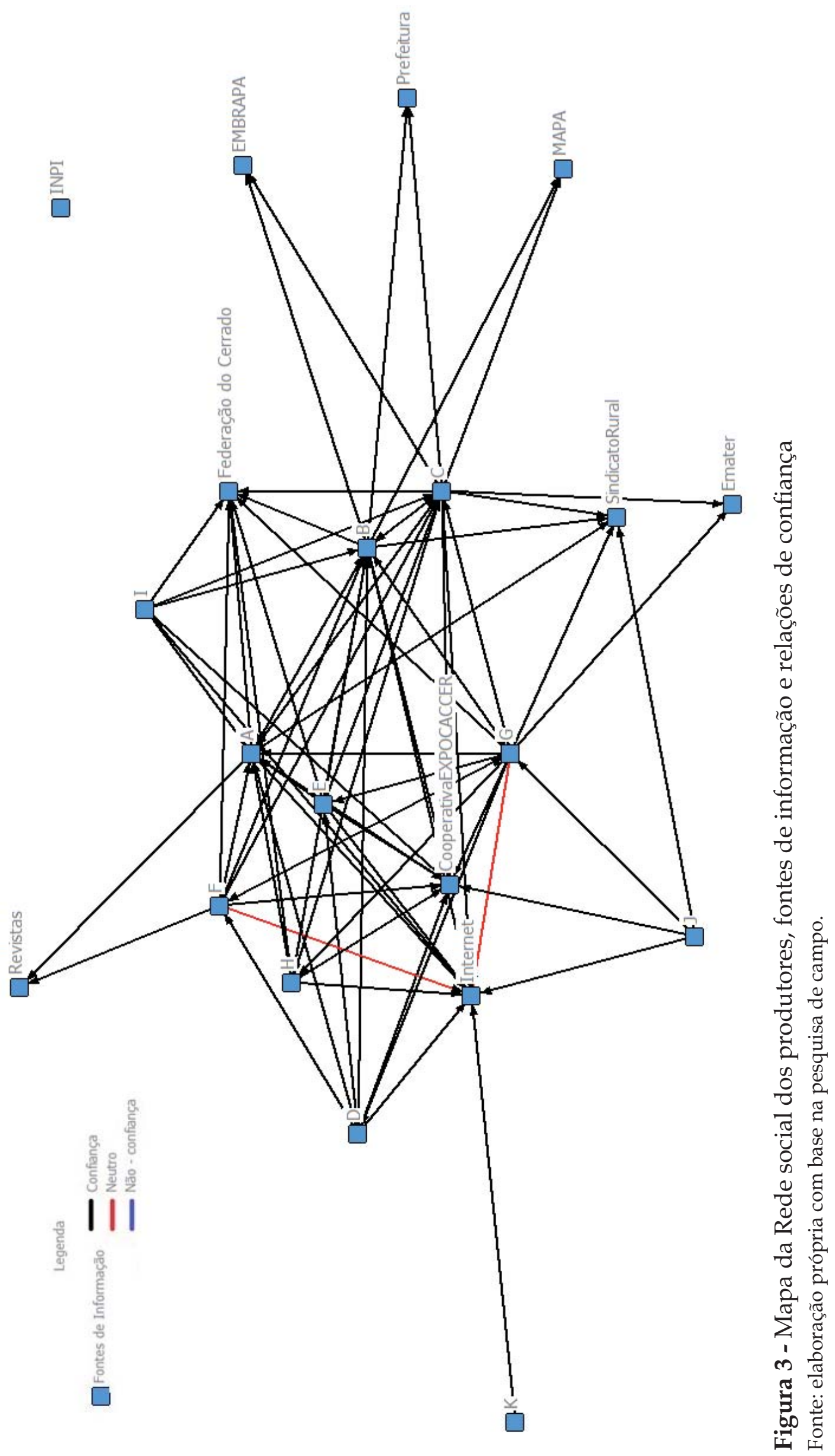


A visualização do mapa permite perceber, primeiramente, que os laços são predominantemente declarados como de confiança, com exceção do uso da internet, em que os produtores $\mathrm{G}$ e $\mathrm{F}$ declararam possuir nível de confiança neutra. Esses produtores se declararam neutros devido à necessidade de selecionar as informações na web, e afirmaram que existem informações falsas e errôneas em diversos portais.

Não houve ocorrência de laços não confiáveis por parte dos produtores rurais.

A comparação das redes de confiança e frequência mostra que, para esse caso, independente da frequência no uso das fontes, existe a confiança por parte dos produtores rurais. Um exemplo pode ser atribuído à Federação dos Cafeicultores do Cerrado, como uma importante fonte de informação, que é utilizada de maneira predominantemente esporádica ou mensal, mas é percebida como confiável por todos os produtores rurais, com exceção do produtor $K$, que não possui relação.

\section{CONSIDERAÇÕES FINAIS}

Os resultados demonstraram que alguns produtores que comercializam com o selo de origem ocupam de fato um papel central, tanto na frequência de busca da informação, como em ser fonte de informação por parte de outros produtores para objetivos ligados a produto, processo produtivo e processos de gestão. Entretanto não é possível generalizar essa afirmativa para todos os produtores que possuam o selo de origem, uma vez que existem unidades produtoras sem selo de origem que obtiveram maior frequência de uso de fonte de informação em relação a produtores com DO, apesar do ritmo menos intenso de adoção de inovações. Isso ocorreu possivelmente porque os produtores sem o selo de origem que obtiveram pontuação elevada objetivam iniciar a comercialização com Selo de Origem assim que conseguirem adequar suas produções.
Este artigo vem contribuir com um tema extremamente importante para os sistemas agroindustriais e demais sistemas, que é o de redes sociais para troca de informação para a inovação, potencializando-se a forma de usá-las para outros segmentos produtivos. E ainda, trazer a análise a um objeto de estudo que obteve avanços de valorização do território local, como a de DO no Cerrado Mineiro, cujo processo de inovação, informação e redes sociais foram cruciais.

\section{REFERÊNCIAS}

AHUJA, G.; SODA, G.; ZAHEER, A. The genesis and dynamic of organizational dynamics. Organization Science, v. 23, n. 2, p. 434-448, 2012.

AHUJA, G.; POLIDORO JR., F.; MITCHELL, W. Structural homophily or social asymmetry? The formation of alliances by poorly embedded firms. Strategic Management Journal, v. 30, n. 9, p. 941-958, 2009.

ALBAGLI, S.; MACIEL, M. L. Capital social e empreendedorismo local. In: LASTRES, $\mathrm{H}$. M. M. et al. Políticas para Promoção de Sistemas Produtivos Locais de MPME. Universidade Federal do Rio de Janeiro, 2002. Disponível em: <http://www.redesist.ie.ufrj.br/nts/ nt33/F223_SaritaMLucia.PDF>. Acesso em: 11 out. 2015.

ALBAGLI, S.; MACIEL, M. L. Informação e conhecimento na inovação e no desenvolvimento local. Ciência da Informação, Brasília, v. 33, n. 3, p.9-16, dez. 2004.

BEAULIEU, C. M. G. Dos sistemas nacionais de pesquisa agrícola aos sistemas nacionais de inovação agrícola: a inserção dos institutos nacionais de pesquisa agropecuária. 2013. 224 p. Tese (Doutorado em Política Científica e Tecnológica) - Instituto de Geociências, Universidade Estadual de Campinas (Unicamp), Campinas, SP, 2013.

BORGATTI, S. P.; EVERETT, M. G.; FREEMAN, L. C. Ucinet for Windows: Software for Social Network Analysis. Harvard, MA: Analytic Technologies, 2002. Disponível em: <https:/ / docs.google.com/viewer?a=v\&pi $\mathrm{d}=$ sites\&srcid=ZGVmYXVsdGRvbWFpbnx hbmFseXppbmdzb2NpYWxuZXR3b3Jrc3xn 
eDoyY2QwMmZiYmJjNzQ0YTFi>. Acesso em: 25 ago. 2015.

BRASIL. Café, saiba mais. 2014. Disponível em: <http://www.agricultura.gov.br/vegetal/ culturas/cafe/saiba-mais>. Acesso em: 28 nov. 2014.

BRUNORI, G.; ROSSI, A.; GUIDI, F. On the New Social Relations around and beyond Food. Analysing Consumers' Role and Action in Gruppi di Acquisto Solidale (Solidarity Purchasing Groups). Sociologia Ruralis, v. 52, n. 1, p. 1-27, 2012.

CASSIOLATO, J. E.; SZAPIRO, Marina. Uma caracterização de arranjos produtivos locais de micro e pequenas empresas. In: LASTRES, H. M. M.; CASSIOLATO, J. E.; MACIEL, M. L. (Org.). Pequena empresa: cooperação e desenvolvimento local. Rio de Janeiro: Relume-Dumará, 2003.

CASSIOLATO, J. E.; LASTRES, H. M. M. Sistemas de inovação e desenvolvimento. As implicações de política. São Paulo em Perspectiva, v. 19, n. 1, p. 34-45, 2005.

COMPANHIA NACIONAL DE ABASTECIMENTO (CONAB). Levantamentos de safra. Disponível em: <http://www.conab.gov. br/conteudos.php?a=1253\&>. Acesso em: mar. 2015.

COOKE, P.; URANGA, M. G.; ETXEBARRIA, G. Regional innovation systems: Institutional and organizational dimensions. Research Policy, v. 26, n. 4, p. 475-491, 1997.

DALFOVO, M. S.; LANA, R. A.; SILVEIRA, A. Métodos quantitativos e qualitativos: um resgate teórico. Revista Interdisciplinar Científica Aplicada, Blumenau, SC, v. 2, n. 4, p. 01-13, 2008.

DOCKS, A. C; TISENKOPFS, T; BOCK, B. B. The concept of agricultural knowledge and innovation systems. In: EU SCAR. Agricultural knowledge and innovation systems in transition - a reflection paper. Bruxelas, 2012. Disponível em: <http://ec.europa.eu/research / bioeconomy/pdf/ki3211999enc_002.pdf>. Acesso em: 19 maio 2014.

EMPRESA DE ASSISTÊNCIA TÉCNICA E EXTENSÃORURAL DOESTADODEMINAS GERAIS (EMATER-MG). Missão e visão. [s.d.]. Disponível em: <http://www.emater. $\mathrm{mg} . \mathrm{gov} \cdot \mathrm{br} /$ portal.cgi?flagweb=site_tpl_
paginas_internas2\&id=6\#.VMsDhmjF8mM>. Acesso em: jan. 2015.

EMPRESA BRASILEIRA DE PESQUISA AGROPECUÁRIA (EMBRAPA). Quem somos. [s.d.]. Disponível em: <https://www.embrapa.br/quem-somos>. Acesso em: jan. 2015.

FREEMAN, C. Networks of innovators: a synthesis of research issues. Research Policy, v. 20, n. 5, p. 499-514, out. 1991.

GRANOVETTER, M. Ação econômica e estrutura social: o problema da imersão. RAEEletrônica, v. 6, n. 1, art. 9, 2007.

GUIMARÃES FILHO, C. Certificação de Indicação Geográfica. Uma estratégia de inserção no mercado para produtos do Semiárido. Secretaria da Agricultura, Pecuária, Irrigação, Reforma Agrária, Pesca e Aquicultura do Estado da Bahia, 2013. Disponível em: <http:/ / www.agricultura.gov.br/desenvolvimentosustentavel/indicacao-geografica>. Acesso em: 13 ago. 2014.

HOLSTER, H.; HORAKOVA, S.; IPEMA, B.; FUSAI, B.; GIANNERINI, G.; TEYE, F.; MARTINI, D.; SHALLOO, L.; SCHMID, O. Current situation on data exchange in agriculture in the EU-27 and Switzerland. AgriXchange project, network for data exchange in agriculture. 2012. Disponível em: <http:/ / edepot.wur. nl/206268>. Acesso em: 6 dez. 2014.

INSTITUTONACIONAL DEPROPRIEDADE INDUSTRIAL (INPI). Revista de propriedade Industrial, seção I, n. 2243, 31 dez. 2013. Disponível em: <http:/ / revistas.inpi.gov.br/ rpi/>. Acesso em: 12 jun. 2014.

JESPERSEN, L. M.; HANSEN, J. P.; BRUNORI, G.; JENSEN, A. L.; HOLST, K.; MATHIESEN, C.; HALBERG, N.; RASMUSSEN, I. A. Ict and social media as drivers of multi-actor innovation in agriculture. Barriers, recommendation and potentials. EU SCAR. Agricultural Knowledge and Innovation Systems Towards - an orientation paper on linking innovation and research 2020. Bruxelas, 2014. Disponível em: <http://ec.europa.eu/ research/bioeconomy/pdf/ki3211999enc_002. pdf>. Acesso em: 19 out. 2014.

MAFRA, L. A. S. Indicação geográfica e construção do mercado: a valorização da origem no Cerrado Mineiro. 2008. 137 p. Tese (Doutorado em Ciências) - Instituto de Ciências Humanas e Sociais, Universidade 
Federal Rural do Rio de Janeiro (UFRRJ), Rio de Janeiro, 2008.

NASCIMENTO, C. L.; JIMENEZ, G. G.; CAMPOMAR, M. C. A rede social como fonte de decisão para o processo de decisão de compra. Future Studies Research Journal, São Paulo, v. 6, n. 2, p. 30-47, 2014.

ORESZCZYN, S.; LANE, A.; CARR, S. The role of networks of practice and webs of influencers on farmersengagement with and learning about agricultural innovations. Journal of Rural Studies, v. 26, n. 4, p. 404-417, out. 2010.

ORGANISATION FOR ECONOMIC CO-OPERATION AND DEVELOPMENT. Statistical Office Of The European Communities. Oslo Manual: Guidelines for collecting and interpreting innovation data. 3 ed., 2005. Disponível em: <http://epp.eurostat.ec.europa.eu/ cache/ITY_PUBLIC/OSLO/EN/OSLO-EN. PDF>. Acesso em: 12 maio 2014.

ORTEGA, A. C.; JESUS, M. C. Café e território. A cafeicultura no Cerrado Mineiro. Campinas, SP: Alínea, 2012.
Território, certificação de procedência e a busca da singularidade: o caso do Café do Cerrado. Política e Sociedade, v. 10, n. 19, p. 305-330, 2011.

POPPE, K. J. Innovation policy: theory and EU initiatives. In: EU SCAR. Agricultural knowledge and innovation systems in transition a reflection paper. Bruxelas, 2012. Disponível em: <http:/ / ec.europa.eu/research / bioeconomy/pdf/ki3211999enc_002.pdf $>$. Acesso em: 19 maio 2014.

SCHAEFER, C. Motivations and usage patterns on social network sites. European Conference on Information Science, Karlsruhe, Alemanha, 2008. Disponível em: <https://pdfs. semanticscholar.org/7bfb/f38d2fcb6996bb1acb0b2bb3dc042d4e4d80.pdf?_ga=1.1739 00963.1222369864.1479999772>.

VOSS, C.; TSIKRIKTSIS, N.; FROLICH, M. Case research in operations management. International Journal of Operations \& Production Management, Londres, v. 22, n. 2, p. 195-219, 2002.

\section{Sobre os autores:}

Douglas Ken Nagai: Programa de Pós-Graduação em Agronegócio e Desenvolvimento PGAD/ Unesp, Campus de Tupã. Centro de Pesquisas em Administração e Agronegócio (CEPEAGRO). Possui graduação em Administração pela Universidade Estadual Paulista Júlio de Mesquita Filho. Mestrando do Programa de Pós-Graduação em Agronegócio e Desenvolvimento da Universidade Estadual Paulista Júlio de Mesquita Filho. Atua na linha de pesquisa de Competitividade de Sistemas Agroindustriais e faz parte do grupo de pesquisa credenciado pelo CNPq, CEPEAGRO (Centro de Pesquisas em Administração e Agronegócio). E-mail: nagaikd@gmail.com

Giuliana Aparecida Santini Pigatto: Programa de Pós-Graduação em Agronegócio e Desenvolvimento PGAD/Unesp, Campus de Tupã. Centro de Pesquisas em Administração e Agronegócio (CEPEAGRO). Possui graduação em Ciências Econômicas pela Universidade Estadual Paulista Júlio de Mesquita Filho (1999), mestrado em Engenharia de Produção pela Universidade Federal de São Carlos (2002) e doutorado em Engenharia de Produção pela Universidade Federal de São Carlos (2006). É docente em Regime de Dedicação Integral à Docência e Pesquisa (RDIDP) pela Universidade Estadual Paulista, Campus de Tupã (CET); Professora permanente do Programa de Pós-graduação em Agronegócio e Desenvolvimento da UNESP de Tupã (área Interdisciplinar Capes); Pesquisadora do CEPEAGRO (Centro de Pesquisa em Administração e Agronegócios). E-mail: giusantini@tupa.unesp.br

Timóteo Ramos Queiroz: Programa de Pós-Graduação em Agronegócio e Desenvolvimento PGAD/Unesp, Campus de Tupã. Centro de Pesquisas em Administração e Agronegócio (CEPEAGRO). Professor Doutor da UNESP, Administrador pela UFMS, Mestre e Doutor em Engenharia de Produção pela UFSCar. Atua como Docente Orientador do Programa de Pós-Graduação em Agronegócio e Desenvolvimento (PGAD/UNESP) e pesquisador do CEPEAGRO/UNESP. E-mail: timoteo@tupa.unesp.br 